\title{
A Lazer-Leach-type condition for singular differential equations with a deviating argument at resonance
}

$\operatorname{Jin} \mathrm{Li}^{*}$

"Correspondence: Ixjin25@163.com School of Science, Jiujiang University, Jiujiang, 332005, China

\begin{abstract}
In this paper, we establish a Lazer-Leach-type condition depending on the delay for the existence of positive periodic solutions for singular differential equations with a deviating argument at resonance. The proof of the main result is based on the phase-plane analysis and topological degree methods.
\end{abstract}

MSC: $34 \mathrm{C} 25 ; 34 \mathrm{D} 40$

Keywords: periodic solutions; deviating argument; singular; at resonance; Lazer-Leach-type condition

\section{Introduction}

In this paper, we deal with the following delay differential equations at resonance:

$$
x^{\prime \prime}+\frac{1}{4} n^{2} x+g(x(t), x(t-\tau))=p(t)
$$

where $g:(0, \infty) \times \mathbb{R} \rightarrow \mathbb{R}$ is continuous, $\tau \geq 0$ is a constant, $p(t)$ is continuous and $2 \pi$ periodic, and the function $g$ has a singularity of repulsive type at the origin for its first variable, that is, $\lim _{s_{1} \rightarrow \infty} g\left(s_{1}, s_{2}\right)=-\infty$.

The periodic problems of singular differential equations had attracted the attentions of many researchers during more than the last two decades because of their background in applied science [1-17]. A landmark work on mathematical treatment of the differential equations with singularities, as we all know, is done by Lazer and Solimini [11]. From then on, some classical mathematical tools were used successfully to study these singular equations, such as Mawhin's continuation theorem in the coincidence degree theory [17], the method of upper and lower solutions [8], some fixed point theorems in cones [2], the Poincaré-Birkhoff theorem [14], the phase-plane analysis and topological degree methods [12], and so on.

Wang and $\mathrm{Ma}[14]$ first studied the resonant singular equation

$$
x^{\prime \prime}+\frac{1}{4} n^{2} x+g(x)=p(t)
$$

(c) The Author(s) 2017. This article is distributed under the terms of the Creative Commons Attribution 4.0 International License (http://creativecommons.org/licenses/by/4.0/), which permits unrestricted use, distribution, and reproduction in any medium, provided you give appropriate credit to the original author(s) and the source, provide a link to the Creative Commons license, and indicate if changes were made. 
where $g$ has a singularity and satisfies

$$
\lim _{x \rightarrow \infty} g(x)=g(+\infty)
$$

They obtained the existence of $2 \pi$-periodic solutions of (1.2) under the following so-called Lazer-Leach-type condition:

$$
4 g(+\infty)-\int_{0}^{2 \pi} p(t)\left|\sin \left(\theta+\frac{n t}{2}\right)\right| d t \neq 0 \quad \text { for all } \theta \in \mathbb{R}
$$

Note that they perfectly answered the open problem raised by del Pino and Manasevich [4].

After that, Wang [18] discussed the periodic problem of the resonant Liénard equation with constant delay but without singularity and established some Lazer-Leach-type conditions depending on the delay. A natural and delicate idea is that the delay may affect the existence of periodic solutions not only for the equations in [18], but also for many other kinds of equations. Based on this, in the present paper, we consider equation (1.1) and look for Lazer-Leach-type conditions. The main difficulty to overcome is the coexistence of the singularity and delay. A possible way for us is to use the phase-plane analysis and topological degree methods, also used in [12], and give the following fundamental hypotheses.

$\left(\mathrm{H}_{1}\right)$ The variables of $g$ are separable, that is, there exist two functions $g_{0}$ and $g_{1}$ such that $g(x(t), x(t-\tau))=g_{0}(x(t))+g_{1}(x(t-\tau))$. Moreover, $g_{0}$ is bounded on $[1,+\infty)$, $\lim _{x \rightarrow+\infty} g_{0}(x)=0$, and $\lim _{x \rightarrow+\infty} g_{1}(x)=g(+\infty)$ is finite.

$\left(\mathrm{H}_{2}\right)$ For all $\theta \in \mathbb{R}$,

$$
4 \cos \frac{n \tau}{2} g(+\infty) \neq \int_{0}^{2 \pi} p(t)\left|\sin \left(\frac{n}{2} t+\theta\right)\right| d t
$$

$\left(\mathrm{H}_{3}\right)$ Put $G_{0}(x)=\int_{1}^{x} g_{0}(s) d s$. It satisfies

$$
\lim _{x \rightarrow 0^{+}} g_{0}(x)=-\infty, \quad \lim _{x \rightarrow 0^{+}} G_{0}(x)=+\infty
$$

Furthermore, there exist $0<\varepsilon_{0} \leq 1,0<l \leq 1$, and $0<L<\infty$ such that

$$
\frac{-g_{0}(x)}{G_{0}^{1+l}(x)}>L \quad \text { for all } x \in\left(0, \varepsilon_{0}\right)
$$

We now state our main theorem.

Theorem 1.1 Let $\left(\mathrm{H}_{1}\right),\left(\mathrm{H}_{2}\right)$, and $\left(\mathrm{H}_{3}\right)$ hold. Then (1.1) has at least one periodic solution.

Remark 1.2 $\left(\mathrm{H}_{3}\right)$ is a universal strong singularity condition. We give some examples to show that $\left(\mathrm{H}_{3}\right)$ can be satisfied.

- $g_{0}(x)=-\frac{1}{x}$. Then $G_{0}(x)=-\ln x$, and (1.5) holds. Let $\varepsilon_{0}=e^{-2}$ and $l=L=1$. By using a short calculation we can obtain that (1.6) holds.

- $g_{0}(x)=-\frac{1}{x^{3}}$. Then $G_{0}(x)=\frac{1}{2 x^{2}}-\frac{1}{2}$, and (1.5) holds. Let $\varepsilon_{0}=L=1$ and $l=\frac{1}{2}$. We can also check that (1.6) holds. 
- $g_{0,1}(x)+g_{0,2}(x)$, where the functions $g_{0, i}(x)(i=1,2)$ satisfy $(1.5)$ and (1.6). In fact, it is easy to see that (1.5) holds for $g_{0,1}(x)+g_{0,2}(x)$ and $G_{0,1}(x)+G_{0,2}(x)$, where $G_{0, i}(x)=\int_{1}^{x} g_{0, i}(s) d s(i=1,2)$. On the other hand, we assume that there exist $0<\varepsilon_{0, i} \leq 1,0<l_{i} \leq 1$, and $0<L_{i} \leq \infty$ such that

$$
\frac{-g_{0, i}(x)}{G_{0, i}^{1+l}(x)}>L_{i} \quad \text { for all } x \in\left(0, \varepsilon_{0, i}\right)
$$

Without loss of generality, we assume also that $G_{0, i}(x)>1$ and $g_{0, i}(x)<-1$ for all $x \in\left(0, \varepsilon_{0, i}\right)$. Set $\varepsilon_{0}=\min \left\{\varepsilon_{0,1}, \varepsilon_{0,2}\right\}$. For all $x \in\left(0, \varepsilon_{0}\right)$, if $G_{0,1}(x)>G_{0,2}(x)$, then

$$
\frac{-g_{0,1}(x)-g_{0,2}(x)}{\left[G_{0,1}(x)+G_{0,2}(x)\right]^{1+l}}>\frac{-g_{0,1}(x)}{\left[2 G_{0,1}(x)\right]^{1+l}}>\frac{L_{1}}{2^{1+l}}
$$

otherwise,

$$
\frac{-g_{0,1}(x)-g_{0,2}(x)}{\left[G_{0,1}(x)+G_{0,2}(x)\right]^{1+l}}>\frac{-g_{0,2}(x)}{\left[2 G_{0,2}(x)\right]^{1+l}}>\frac{L_{2}}{2^{1+l}} .
$$

Hence (1.6) holds.

Remark 1.3 When $\tau=0$, condition (1.4) degenerates to condition (1.3). Therefore Theorem 1.1 generalizes the result in [14]. Moreover, the delay $\tau$ may affect the existence of periodic solutions.

This paper is structured into three sections. Section 2 is devoted to the proof of a useful lemma. In Section 3, we state some lemmas to prove the main theorem.

\section{Preliminary lemma}

To use the phase-plane analysis and topological degree methods, we embed (1.1) into a family of equations with one parameter $\lambda \in[0,1]$,

$$
x^{\prime \prime}+\frac{1}{4} n^{2} x+(1-\lambda)\left(-1-\frac{1}{x^{3}}\right)+\lambda g(x(t), x(t-\tau))=\lambda p(t) .
$$

Now, we give the following fundamental lemma.

Lemma 2.1 Suppose that there exist three positive constants $M_{0}, M_{1}$, and $M_{2}$ such that, for any $2 \pi$-periodic solution $x(t)$ of $(2.1)$,

$$
M_{0}<x(t)<M_{1} \quad \text { for all } t \in \mathbb{R}
$$

and

$$
\left\|x^{\prime}\right\|_{\infty} \triangleq \max _{t \in[0,2 \pi]}\left|x^{\prime}(t)\right|<M_{2}
$$

Then Eq. (1.1) has at least one $2 \pi$-periodic solution.

Since the proof is similar as that in [12], we omit it. 
Remark 2.2 In fact, Lemma 2.1 is also valid if we embed (1.1) into the following family of equations with one parameter $\lambda \in[0,1]$ :

$$
x^{\prime \prime}+\frac{1}{4} n^{2} x+(1-\lambda)\left(1-\frac{1}{x^{3}}\right)+\lambda g(x(t), x(t-\tau))=\lambda p(t)
$$

\section{The proof of the main theorem}

Condition $\left(\mathrm{H}_{2}\right)$ reduces to

$\left(\mathrm{H}_{2}^{\prime}\right)$ for all $\theta \in \mathbb{R}$,

$$
4 \cos \frac{n \tau}{2} g(+\infty)<\int_{0}^{2 \pi} p(t)\left|\sin \left(\frac{n}{2} t+\theta\right)\right| d t
$$

or

$\left(\mathrm{H}_{2}^{\prime \prime}\right)$ for all $\theta \in \mathbb{R}$,

$$
4 \cos \frac{n \tau}{2} g(+\infty)>\int_{0}^{2 \pi} p(t)\left|\sin \left(\frac{n}{2} t+\theta\right)\right| d t
$$

In this section, we always assume that $\left(\mathrm{H}_{2}^{\prime}\right)$ holds. The argument for $\left(\mathrm{H}_{2}^{\prime \prime}\right)$ is similar.

We first suppose that the sequence $\left\{\left(x_{k}, y_{k}\right)\right\}_{k=1}^{\infty}$ satisfies

$$
x_{k}^{\prime}=y_{k}, \quad y_{k}^{\prime}=-\frac{1}{4} n^{2} x_{k}-g\left(x_{k}, x_{k}(t-\tau), \lambda_{k}\right)
$$

with $\left\|x_{k}\right\|_{\infty}+\left\|y_{k}\right\|_{\infty} \rightarrow \infty$ as $k \rightarrow \infty$, where

$$
g\left(x_{k}, x_{k}(t-\tau), \lambda_{k}\right)=\left(1-\lambda_{k}\right)\left(-1-\frac{1}{x_{k}^{3}}\right)+\lambda_{k} g_{0}\left(x_{k}\right)+\lambda_{k} g_{1}\left(x_{k}(t-\tau)\right)-\lambda_{k} p(t)
$$

In this position, we only consider $\lambda_{k} \rightarrow \lambda_{0} \in[0,1]$. Even if $\lambda_{k}$ has no limit, we can consider its convergent subsequence $\left\{\lambda_{k_{i}}\right\}$ and the corresponding solution sequence $\left\{\left(x_{k_{i}}, y_{k_{i}}\right)\right\}$ for (3.1) because the sequence $\left\{\lambda_{k}\right\}$ is bounded. For simplicity, we omit these discussions. It is easy to see that $\left\|x_{k}\right\|_{\infty}+\left\|y_{k}\right\|_{\infty} \rightarrow \infty$ is equivalent to $\left\|x_{k}\right\|_{\infty} \rightarrow \infty$ and $\left\|y_{k}\right\|_{\infty} \rightarrow \infty$ as $k \rightarrow \infty$. Define

$$
g_{0}\left(x_{k}, \lambda_{k}\right)=-\left(1-\lambda_{k}\right) \frac{1}{x_{k}^{3}}+\lambda_{k} g_{0}\left(x_{k}\right)
$$

and

$$
g_{1}\left(x_{k}(t-\tau), \lambda_{k}\right)=-\left(1-\lambda_{k}\right)+\lambda_{k} g_{1}\left(x_{k}(t-\tau)\right) .
$$

Obviously, $g_{0}\left(x_{k}, \lambda_{k}\right)$ satisfies condition $\left(\mathrm{H}_{3}\right)$.

Take the transformation

$$
x_{k}=1+r_{k} \cos \theta_{k}, \quad y_{k}=\frac{n}{2} r_{k} \sin \theta_{k}
$$


Then system (3.1) is equivalent to the following system:

$$
\left\{\begin{array}{l}
\frac{d \theta_{k}}{d t}=-\frac{n}{2}-\frac{2}{n r_{k}} g\left(1+r_{k} \cos \theta_{k}, 1+r_{k}(t-\tau) \cos \theta_{k}(t-\tau), \lambda_{k}\right) \cos \theta_{k}+\frac{n}{2 r_{k}} \cos \theta_{k}, \\
\frac{d r_{k}}{d t}=-\frac{n}{2} \sin \theta_{k}-\frac{2}{n} g\left(1+r_{k} \cos \theta_{k}, 1+r_{k}(t-\tau) \cos \theta_{k}(t-\tau), \lambda_{k}\right) \sin \theta_{k} .
\end{array}\right.
$$

Lemma 3.1 Assume that $\left(\mathrm{H}_{1}\right)$ and $\left(\mathrm{H}_{3}\right)$ hold. For $k$ large enough, we have

$$
\theta_{k}^{\prime}(t)<0 \quad \forall t \in \mathbb{R}
$$

Proof By $\left(\mathrm{H}_{3}\right)$ and the definition of $g\left(x_{k}, x_{k}(t-\tau), \lambda_{k}\right)$ we have that there exists $0<\delta<1$ such that

$$
g\left(x_{k}, x_{k}(t-\tau), \lambda_{k}\right)-\frac{n^{2}}{4}<0
$$

Noticing that $\cos \theta_{k}<0$ for $0<x_{k} \leq \delta<1$, we get, for $0<x_{k} \leq \delta$,

$$
\frac{d \theta_{k}}{d t} \leq-\frac{n}{2}
$$

Since $\left\|x_{k}\right\|_{\infty} \rightarrow \infty$ and $\left\|y_{k}\right\|_{\infty} \rightarrow \infty$ as $k \rightarrow \infty$, we have that if $x_{k}>\delta$, then

$$
r_{k} \rightarrow \infty \quad \text { as } k \rightarrow \infty
$$

Meanwhile, if $x_{k}>\delta$, by $\left(\mathrm{H}_{1}\right)$, then we get that there exists $M>0$ such that

$$
\left|g\left(x_{k}, x_{k}(t-\tau), \lambda_{k}\right)\right|<M
$$

Hence, for $k$ large enough, if $x_{k}>\delta$, then

$$
\left|-\frac{2}{n r_{k}} g\left(1+r_{k} \cos \theta_{k}, 1+r_{k}(t-\tau) \cos \theta_{k}(t-\tau), \lambda_{k}\right) \cos \theta_{k}+\frac{n}{2 r_{k}} \cos \theta_{k}\right|<\frac{n}{4},
$$

and then

$$
\frac{d \theta_{k}}{d t} \leq-\frac{n}{2}+\frac{n}{4}=-\frac{n}{4}
$$

From (3.3) and (3.4) we obtain the conclusion of Lemma 3.1.

From Lemma 3.1 we conclude that, for $k$ large enough, the solution $\left(x_{k}(t), x_{k}(t)\right)$ of (3.1) makes clockwise rotations around the point $(1,0)$. Without loss of generality, we take the initial point $\left(x_{k}\left(t_{0}^{m, k}\right), y_{k}\left(t_{0}^{m, k}\right)\right)$ of the $m$ th rotation that satisfies

$$
x_{k}\left(t_{0}^{m, k}\right)=1, \quad y_{k}\left(t_{0}^{m, k}\right)=x_{k}^{\prime}\left(t_{0}^{m, k}\right)>0
$$

and

$$
\theta_{k}\left(t_{0}^{m, k}\right)=\frac{\pi}{2}-2(m-1) \pi,
$$

where $m=1,2, \ldots$. 
Let $\left(x_{k}(t), y_{k}(t)\right)$ take exactly one rotation from $t_{0}^{m, k}$. Then there are two points where the curve $\left(x_{k}(t), y_{k}(t)\right)$ meets the line $x_{k}=1$ and two points where the curve $\left(x_{k}(t), y_{k}(t)\right)$ meets the $x_{k}$-axis. We denote them by $\left(x_{k}\left(t_{1}^{m, k}\right), 0\right),\left(1, y_{k}\left(t_{2}^{m, k}\right)\right),\left(x_{k}\left(t_{3}^{m, k}\right), 0\right)$, and $\left(1, y_{k}\left(t_{4}^{m, k}\right)\right)$, where $x_{k}\left(t_{1}^{m, k}\right)>1, y_{k}\left(t_{2}^{m, k}\right)<0,0<x_{k}\left(t_{3}^{m, k}\right)<1$, and $y_{k}\left(t_{4}^{m, k}\right)=y_{k}\left(t_{0}^{m+1, k}\right)>0$.

Define

$$
\mathcal{R}_{k}^{2}(t)=\left(x_{k}(t)-1\right)^{2}+\frac{4}{n^{2}} y_{k}^{2}(t)+\int_{t_{0}^{1, k}}^{t} \frac{8}{n^{2}} y_{k}(s)\left(g\left(x_{k}(s), x_{k}(s-\tau), \lambda_{k}\right)+\frac{1}{4} n^{2}\right) d s .
$$

Since $\frac{d \mathcal{R}_{k}^{2}}{d t} \equiv 0$, we get $\mathcal{R}_{k}^{2}(t)=\mathcal{R}_{k}^{2}$, where $\mathcal{R}_{k}>0$. Obviously, we also get that

$$
\mathcal{R}_{k}^{2}(t)=x_{k}^{2}(t)+\frac{4}{n^{2}} y_{k}^{2}(t)+\int_{t_{0}^{1, k}}^{t} \frac{8}{n^{2}} y_{k}(s) g\left(x_{k}(s), x_{k}(s-\tau), \lambda_{k}\right) d s-1
$$

and define

$$
G_{k}(t)=\int_{t_{0}^{1, k}}^{t} \frac{8}{n^{2}} y_{k}(s)\left(g\left(x_{k}(s), x_{k}(s-\tau), \lambda_{k}\right)+\frac{1}{4} n^{2}\right) d s .
$$

Therefore we have $\mathcal{R}_{k}^{2}=r_{k}^{2}(t)+G_{k}(t)$.

Lemma 3.2 Assume that $\left(\mathrm{H}_{1}\right)$ and $\left(\mathrm{H}_{3}\right)$ hold. Then

$$
\lim _{k \rightarrow \infty} x_{k}\left(t_{1}^{m, k}\right)=+\infty \text { and } \lim _{k \rightarrow \infty} x_{k}\left(t_{3}^{m, k}\right)=0
$$

for $m=1,2, \ldots$

Proof Without loss of generality, we assume that there exist a positive integer $m^{*}$ such that

$$
x_{k}\left(t_{1}^{m^{*}, k}\right)=\max _{t \in \mathbb{R}} x_{k}(t)
$$

Noticing that $\lim _{k \rightarrow \infty} \max _{t \in \mathbb{R}} x_{k}(t) \rightarrow \infty$, we get $\lim _{k \rightarrow \infty} x_{k}\left(t_{1}^{m^{*}, k}\right) \rightarrow \infty$. We will prove that $\lim _{k \rightarrow \infty} x_{k}\left(t_{3}^{m^{*}, k}\right)=0$ and $\lim _{k \rightarrow \infty} x_{k}\left(t_{1}^{m^{*}+1, k}\right)=+\infty$, and the others are similar.

We first prove that $\lim _{k \rightarrow \infty} x_{k}\left(t_{3}^{m^{*}, k}\right)=0$. Assume by contradiction that there exists a constant $0<c<1$ such that $x_{k}\left(t_{3}^{m^{*}, k}\right)>c$ for $k \in \mathbb{N}^{+}$. Thus, if $t \in\left[t_{1}^{m^{*}, k}, t_{3}^{m^{*}, k}\right]$, then $g\left(x_{k}, x_{k}(t-\tau), \lambda_{k}\right)$ is bounded. Hence, from the second equality of (3.2) we obtain $r_{k}\left(t_{3}^{m^{*}, k}\right)=$ $r_{k}\left(t_{1}^{m^{*}, k}\right)+O(1)$. Therefore, $r_{k}\left(t_{3}^{m^{*}, k}\right) \rightarrow \infty$, which contradicts the fact $0<x_{k}\left(t_{3}^{m^{*}, k}\right)<1$ and $x_{k}^{\prime}\left(t_{3}^{m^{*}, k}\right)=0$. Consequently, $\lim _{k \rightarrow \infty} x_{k}\left(t_{3}^{m^{*}, k}\right)=0$.

Next, we prove that $\lim _{k \rightarrow \infty} x_{k}\left(t_{1}^{m^{*}+1, k}\right)=+\infty$. Since $g\left(x_{k}, x_{k}(t-\tau), \lambda_{k}\right)$ is bounded for $t \in\left[t_{1}^{m^{*}, k}, t_{2}^{m^{*}, k}\right]$, we have $\lim _{k \rightarrow \infty} r_{k}\left(t_{2}^{m^{*}, k}\right) \rightarrow \infty$. It follows from $x_{k}\left(t_{2}^{m^{*}, k}\right)=1$ that $\lim _{k \rightarrow \infty} y_{k}\left(t_{2}^{m^{*}, k}\right) \rightarrow \infty$. By (3.6) we obtain

$$
\begin{aligned}
G_{k}\left(t_{0}^{m^{*}+1, k}\right)-G_{k}\left(t_{2}^{m^{*}, k}\right) & =G_{k}\left(t_{4}^{m^{*}, k}\right)-G_{k}\left(t_{2}^{m^{*}, k}\right) \\
& =\int_{t_{2}^{m^{*}, k}}^{t_{4}^{m^{*}, k}} \frac{8}{n^{2}} y_{k}(s)\left(g\left(x_{k}(s), x_{k}(s-\tau), \lambda_{k}\right)+\frac{1}{4} n^{2}\right) d s
\end{aligned}
$$




$$
\begin{aligned}
& =\int_{t_{2}^{m^{*}, k}}^{t_{4}^{m^{*}, k}} \frac{8 \lambda_{k}}{n^{2}} y_{k}(s) g_{1}\left(x_{k}(s-\tau)\right) d s \\
& =o\left(r_{k}^{2}\left(t_{2}^{m^{*}, k}\right)\right) .
\end{aligned}
$$

It follows from the relation $\mathcal{R}_{k}^{2}=r_{k}^{2}(t)+G_{k}(t)$ that

$$
r_{k}^{2}\left(t_{0}^{m^{*}+1, k}\right)=r_{k}^{2}\left(t_{2}^{m^{*}, k}\right)-\left(G_{k}\left(t_{0}^{m^{*}+1}\right)-G_{k}\left(t_{2}^{m^{*}, k}\right)\right)=r_{k}^{2}\left(t_{2}^{m^{*}, k}\right)+o\left(r_{k}^{2}\left(t_{2}^{m^{*}, k}\right)\right) .
$$

Hence, $r_{k}\left(t_{0}^{m^{*}+1, k}\right) \rightarrow \infty$. By using the second equality of (3.2) again we obtain $r_{k}\left(t_{1}^{m^{*}+1, k}\right) \rightarrow$ $\infty$. Equivalently, $\lim _{k \rightarrow \infty} x_{k}\left(t_{1}^{m^{*}+1, k}\right)=+\infty$.

Denote by $\tau_{k}^{m}$ the required time for the solution $\left(x_{k}(t), y_{k}(t)\right)$ to complete the $m$ th rotation around the point $(0,1)$. Then,

$$
\tau_{k}^{m}=\left(t_{2}^{m, k}-t_{0}^{m, k}\right)+\left(t_{4}^{m, k}-t_{2}^{m, k}\right) .
$$

Lemma 3.3 Assume that $\left(\mathrm{H}_{1}\right)$ and $\left(\mathrm{H}_{3}\right)$ hold. Then, for $k$ large enough,

$$
\tau_{k}^{m}=\frac{2 \pi}{n}+o(1)
$$

Proof We first compute $t_{2}^{m, k}-t_{0}^{m, k}$. Since $g\left(x_{k}, x_{k}(t-\tau), \lambda_{k}\right)$ is bounded on the interval $\left[t_{0}^{m, k}, t_{2}^{m, k}\right]$, we get from the first equality of (3.2) that

$$
\frac{d t}{d \theta_{k}}=-\frac{2}{n}+o(1)
$$

which implies $t_{2}^{m, k}-t_{0}^{m, k}=\frac{2 \pi}{n}+o(1)$.

Next, we estimate $\left(t_{4}^{m, k}-t_{2}^{m, k}\right)$. By (3.5) we have, for $t \in\left[t_{2}^{m, k}, t_{3}^{m, k}\right]$,

$$
\frac{d t}{d x_{k}}=\frac{1}{y_{k}}=-\frac{2}{n} \frac{1}{\sqrt{\mathcal{R}_{k}^{2}-\left(x_{k}(t)-1\right)^{2}-G_{k}(t)}} .
$$

Furthermore,

$$
-\frac{2}{n} d x_{k}=\sqrt{\mathcal{R}_{k}^{2}-\left(x_{k}(t)-1\right)^{2}-G_{k}(t)} d t .
$$

By $\left(\mathrm{H}_{3}\right)$ and Remark 1.2 we have that there exist $0<\varepsilon_{0} \leq 1,0<l \leq 1$, and $0<L<\infty$ such that

$$
\frac{-g_{0}\left(x_{k}, \lambda_{k}\right)}{G_{0}^{1+l}\left(x_{k}, \lambda_{k}\right)}>L \quad \text { for all } x \in\left(0, \varepsilon_{0}\right)
$$

For above $l$, we can choose $t_{-}^{m, k} \in\left[t_{2}^{m, k}, t_{3}^{m, k}\right]$ such that

$$
\begin{aligned}
& G_{k}\left(t_{-}^{m, k}\right)=\mathcal{R}_{k}^{\frac{2}{1+\frac{l}{2}}}, \\
& \mathcal{R}_{k}^{\frac{2}{1+\frac{l}{2}}} \leq G_{k}(t) \leq \mathcal{R}_{k}^{2} \quad \text { for } t \in\left[t_{-}^{m, k}, t_{3}^{m, k}\right],
\end{aligned}
$$


and

$$
G_{k}(t) \leq \mathcal{R}_{k}^{\frac{2}{1+\frac{l}{2}}} \quad \text { for } t \in\left[t_{2}^{m, k}, t_{-}^{m, k}\right]
$$

Integrating over $\left[t_{2}^{m, k}, t_{-}^{m, k}\right]$ for (3.7), we have

$$
\int_{x_{k}\left(t_{2}^{m, k}\right)}^{x_{k}\left(t_{-}^{m, k}\right)}-\frac{2}{n} d x_{k}=\int_{t_{2}^{m, k}}^{t_{-}^{m, k}} \sqrt{\mathcal{R}_{k}^{2}-\left(x_{k}(t)-1\right)^{2}-G_{k}(t)} d t
$$

Noticing that $x_{k}\left(t_{2}^{m, k}\right) \rightarrow 1$ and $x_{k}\left(t_{-}^{m, k}\right) \rightarrow 0$, we get

$$
\int_{x_{k}\left(t_{2}^{m, k}\right)}^{x_{k}\left(t_{-}^{m, k}\right)}-\frac{2}{n} d x_{k}=\frac{2}{n}\left(1-x_{k}\left(t_{-}^{m, k}\right)\right)=\frac{2}{n}+o(1) .
$$

On the other hand, we get

$$
\begin{aligned}
& \int_{t_{2}^{m, k}}^{t_{-}^{m, k}} \sqrt{\mathcal{R}_{k}^{2}-\left(x_{k}(t)-1\right)^{2}-G_{k}(t)} d t \\
& \quad=\left(t_{-}^{m, k}-t_{2}^{m, k}\right) \int_{0}^{1} \sqrt{\mathcal{R}_{k}^{2}-\left(x_{k}\left(t_{2}^{m, k}+s\left(t_{-}^{m, k}-t_{2}^{m, k}\right)\right)-1\right)^{2}-G_{k}\left(t_{2}^{m, k}+s\left(t_{-}^{m, k}-t_{2}^{m, k}\right)\right)} d s \\
& \quad=\left(t_{-}^{m, k}-t_{2}^{m, k}\right) \mathcal{R}_{k} \int_{0}^{1} \sqrt{1-\frac{\left(x_{k}\left(t_{2}^{m, k}+s\left(t_{-}^{m, k}-t_{2}^{m, k}\right)\right)-1\right)^{2}}{\mathcal{R}_{k}^{2}}-\frac{G_{k}\left(t_{2}^{m, k}+s\left(t_{-}^{m, k}-t_{2}^{m, k}\right)\right)}{\mathcal{R}_{k}^{2}}} d s .
\end{aligned}
$$

Thus we have

$$
\frac{\frac{2}{n}+o(1)}{\left(t_{-}^{m, k}-t_{2}^{m, k}\right) \mathcal{R}_{k}}=\int_{0}^{1} \sqrt{1-\frac{\left(x_{k}\left(t_{2}^{m, k}+s\left(t_{-}^{m, k}-t_{2}^{m, k}\right)\right)-1\right)^{2}}{\mathcal{R}_{k}^{2}}-\frac{G_{k}\left(t_{2}^{m, k}+s\left(t_{-}^{m, k}-t_{2}^{m, k}\right)\right)}{\mathcal{R}_{k}^{2}}} d s .
$$

Passing to limits and using the Lebesgue dominated convergence theorem, we get

$$
\lim _{k \rightarrow \infty} \mathcal{R}_{k}\left(t_{-}^{m, k}-t_{2}^{m, k}\right)=\frac{2}{n}
$$

which implies

$$
t_{-}^{m, k}-t_{2}^{m, k}=\frac{2}{n \mathcal{R}_{k}}+o\left(\frac{1}{\mathcal{R}_{k}}\right)
$$

By (3.1) we get, for $t \in\left[t_{-}^{m, k}, t_{3}^{m, k}\right]$,

$$
\begin{aligned}
\frac{d t}{d y_{k}} & =\frac{1}{-\frac{1}{4} n^{2} x_{k}-g\left(x_{k}, x_{k}(t-\tau), \lambda_{k}\right)} \\
& =\frac{1}{-\frac{1}{4} n^{2} x_{k}-g_{0}\left(x_{k}, \lambda_{k}\right)-g_{1}\left(x_{k}(t-\tau), \lambda_{k}\right)+\lambda_{k} p(t)} \\
& =\frac{1}{-g_{0}\left(x_{k}, \lambda_{k}\right)+O(1)} .
\end{aligned}
$$


Since $x_{k}(t) \rightarrow 0$ as $k \rightarrow \infty$ for $t \in\left[t_{-}^{m, k}, t_{3}^{m, k}\right]$, we obtain, for $k$ large enough, $\left[x_{k}\left(t_{3}^{m, k}\right)\right.$, $\left.x_{k}\left(t_{-}^{m, k}\right)\right] \subset\left(0, \varepsilon_{0}\right)$. Then, for $t \in\left[t_{-}^{m, k}, t_{3}^{m, k}\right]$,

$$
\frac{1}{-g_{0}\left(x_{k}, \lambda_{k}\right)}<\frac{1}{L} \frac{1}{G_{0}^{1+l}\left(x_{k}, \lambda_{k}\right)},
$$

which yields that, for $t \in\left[t_{-}^{m, k}, t_{3}^{m, k}\right]$,

$$
\begin{aligned}
\frac{d t}{d y_{k}} & \leq \frac{1}{L} \frac{1}{G_{0}^{1+l}\left(x_{k}, \lambda_{k}\right)}+o\left(\frac{1}{G_{0}^{1+l}\left(x_{k}, \lambda_{k}\right)}\right) \\
& =\frac{1}{L} \frac{1}{G_{k}^{1+l}(t)}+o\left(\frac{1}{G_{k}^{1+l}(t)}\right) \\
& \leq \frac{1}{L} \frac{1}{\mathcal{R}_{k}^{\frac{2(1+l)}{1+\frac{l}{2}}}}+o\left(\frac{1}{\mathcal{R}_{k}^{\frac{2(1+l)}{1+\frac{l}{2}}}}\right) .
\end{aligned}
$$

Integrating over $\left[t_{-}^{m, k}, t_{3}^{m, k}\right]$ and noticing that $y_{k}(t) \leq \frac{n}{2} \mathcal{R}_{k}$, we have

$$
t_{3}^{m, k}-t_{-}^{m, k} \leq \frac{n}{2 L \mathcal{R}_{k}^{\frac{2(1+l)}{1+\frac{l}{2}}}-1}+o\left(\frac{1}{\mathcal{R}_{k}^{\frac{2(1+l)}{1+\frac{l}{2}}}-1}\right)
$$

which implies that

$$
t_{3}^{m, k}-t_{-}^{m, k}=o\left(\frac{1}{\mathcal{R}_{k}}\right)
$$

It follows from (3.8) and (3.9) that

$$
t_{3}^{m, k}-t_{2}^{m, k}=\frac{2}{n \mathcal{R}_{k}}+o\left(\frac{1}{\mathcal{R}_{k}}\right)
$$

Using a similar argument, we can prove that $t_{4}^{m, k}-t_{3}^{m, k}=\frac{2}{n \mathcal{R}_{k}}+o\left(\frac{1}{\mathcal{R}_{k}}\right)$. It follows that

$$
t_{4}^{m, k}-t_{2}^{m, k}=\frac{4}{n \mathcal{R}_{k}}+o\left(\frac{1}{\mathcal{R}_{k}}\right)
$$

Then $t_{4}^{m, k}-t_{2}^{m, k}=o(1)$. This completes the proof.

Since $\left(x_{k}, y_{k}\right)$ is $2 \pi$-periodic, by Lemma 3.3 we know that, for $k$ sufficiently large, $\left(x_{k}, y_{k}\right)$ makes exactly $n$ clockwise revolutions around the point $(1,0)$ as $t$ varies from 0 to $2 \pi$.

We use the following lemma to estimate the maxima.

Lemma 3.4 Assume that $\left(\mathrm{H}_{1}\right),\left(\mathrm{H}_{2}^{\prime}\right)$, and $\left(\mathrm{H}_{3}\right)$ hold. There exist two positive constants $M_{1}$ and $M_{2}$ such that, for any $2 \pi$-periodic solution $x(t)$ of $E q .(2.1)$,

$$
\|x\|_{\infty}<M_{1}, \quad\left\|x^{\prime}\right\|_{\infty}<M_{2}
$$


Proof Assume by contradiction that there exists a sequence $\left\{\left(x_{k}, y_{k}\right)\right\}_{k=1}^{\infty}$ satisfying the system (3.1) with $\left\|x_{k}\right\|_{\infty}+\left\|y_{k}\right\|_{\infty} \rightarrow \infty$ as $k \rightarrow \infty$. It follows that $\left\|x_{k}\right\|_{\infty} \rightarrow \infty$ and $\left\|y_{k}\right\|_{\infty} \rightarrow \infty$.

Using the transformation

$$
x_{k}=1+r_{k} \cos \theta_{k}, \quad y_{k}=\frac{n}{2} r_{k} \sin \theta_{k}
$$

we have system (3.2).

Without loss of generality, we take the initial point $\left(x_{k}\left(t_{0}^{m, k}\right), y_{k}\left(t_{0}^{m, k}\right)\right)$ of the $m$ th rotation satisfying

$$
x_{k}\left(t_{0}^{m, k}\right)=1, \quad y_{k}\left(t_{0}^{m, k}\right)=x_{k}^{\prime}\left(t_{0}^{m, k}\right)>0
$$

and

$$
\theta_{k}\left(t_{0}^{m, k}\right)=\frac{\pi}{2}-2(m-1) \pi
$$

for $m=1,2, \ldots, n$.

Let $\left(x_{k}(t), y_{k}(t)\right)$ take exactly one rotation from $t_{0}^{m, k}$. Denote by $\tau_{k}$ the required time for the solution $\left(x_{k}(t), y_{k}(t)\right)$ to complete $n$ rotations around the point $(0,1)$. Then,

$$
\tau_{k}=\sum_{m=1}^{n} \tau_{k}^{m}=\sum_{m=1}^{n}\left(t_{4}^{m, k}-t_{0}^{m, k}\right)=\sum_{m=1}^{n}\left(t_{2}^{m, k}-t_{0}^{m, k}\right)+\sum_{m=1}^{n}\left(t_{4}^{m, k}-t_{2}^{m, k}\right) .
$$

We further estimate $\sum_{m=1}^{n}\left(t_{2}^{m, k}-t_{0}^{m, k}\right)$. By the second equality of (3.2) we get, for $t \in$ $\left(t_{0}^{m, k}, t_{2}^{m, k}\right)$,

$$
r_{k}(t)=r_{k}\left(t_{0}^{m}\right)+O(1)
$$

Hence, for all $t \in\left[t_{0}^{m, k}, t_{2}^{m, k}\right], 1 \leq m \leq n$,

$$
r_{k}(t)=\mathcal{R}_{k}+o\left(\mathcal{R}_{k}\right)
$$

Furthermore, $\frac{1}{r_{k}(t)}=\frac{1}{\mathcal{R}_{k}}+o\left(\frac{1}{\mathcal{R}_{k}}\right)$ for $t \in\left[t_{0}^{m, k}, t_{2}^{m, k}\right]$.

By the first equality of (3.2), we obtain, for $t \in\left[t_{0}^{m, k}, t_{2}^{m, k}\right]$,

$$
\begin{aligned}
\frac{d t}{d \theta_{k}}= & -\frac{2}{n} \\
& \cdot \frac{1}{1+\frac{4}{n^{2} \mathcal{R}_{k}} g\left(1+r_{k} \cos \theta_{k}, 1+r_{k}(t-\tau) \cos \theta_{k}(t-\tau), \lambda_{k}\right) \cos \theta_{k}+\frac{1}{\mathcal{R}_{k}} \cos \theta_{k}+o\left(\frac{1}{\mathcal{R}_{k}}\right)} \\
= & -\frac{2}{n}+\frac{8}{n^{3} \mathcal{R}_{k}} g\left(1+r_{k} \cos \theta_{k}, 1+r_{k}(t-\tau) \cos \theta_{k}(t-\tau), \lambda_{k}\right) \cos \theta_{k} \\
& +\frac{2}{n \mathcal{R}_{k}} \cos \theta_{k}+o\left(\frac{1}{\mathcal{R}_{k}}\right) .
\end{aligned}
$$


Integrating over $\left[-\frac{\pi}{2}-2(m-1) \pi, \frac{\pi}{2}-2(m-1) \pi\right]$, we get

$$
\begin{aligned}
t_{2}^{m, k}- & t_{0}^{m, k} \\
= & \int_{-\frac{\pi}{2}-2(m-1) \pi}^{\frac{\pi}{2}-2(m-1) \pi}\left[\frac{2}{n}-\frac{8}{n^{3} \mathcal{R}_{k}} g\left(1+r_{k} \cos \theta_{k}, 1+r_{k}(t-\tau) \cos \theta_{k}(t-\tau), \lambda_{k}\right) \cos \theta_{k}\right. \\
& \left.-\frac{2}{n \mathcal{R}_{k}} \cos \theta_{k}+o\left(\frac{1}{\mathcal{R}_{k}}\right)\right] d \theta_{k} \\
= & \frac{2 \pi}{n}-\frac{4}{n \mathcal{R}_{k}}-\int_{-\frac{\pi}{2}-2(m-1) \pi}^{\frac{\pi}{2}-2(m-1) \pi} \frac{8}{n^{3} \mathcal{R}_{k}} \\
& \cdot g\left(1+r_{k} \cos \theta_{k}, 1+r_{k}(t-\tau) \cos \theta_{k}(t-\tau), \lambda_{k}\right) \cos \theta_{k} d \theta_{k}+o\left(\frac{1}{\mathcal{R}_{k}}\right) .
\end{aligned}
$$

Recalling that $\lambda_{k} \rightarrow \lambda_{0} \in[0,1]$, we distinguish two cases.

Case 1: $\lambda_{0}=0$. In this case, recalling that $g_{0}\left(x_{k}\right)+g_{1}\left(x_{k}(t-\tau)\right)-p(t)$ is bounded for $\theta_{k} \in\left(-\frac{\pi}{2}-2(m-1) \pi, \frac{\pi}{2}-2(m-1) \pi\right)$, we have, for $k$ large enough,

$$
\left|\lambda_{k} g_{0}\left(x_{k}\right)+\lambda_{k} g_{1}\left(x_{k}(t-\tau)\right)-\lambda_{k} p(t)\right|<\frac{1}{3}
$$

Then it follows from the definition of $g\left(1+r_{k} \cos \theta_{k}, 1+r_{k}(t-\tau) \cos \theta_{k}(t-\tau), \lambda_{k}\right)$ that, for $k$ large enough (such that $\lambda_{k}<\frac{1}{3}$ ) and for $\theta_{k} \in\left(-\frac{\pi}{2}-2(m-1) \pi, \frac{\pi}{2}-2(m-1) \pi\right)$,

$$
g\left(1+r_{k} \cos \theta_{k}, 1+r_{k}(t-\tau) \cos \theta_{k}(t-\tau), \lambda_{k}\right)<-\left(1-\lambda_{k}\right)+\frac{1}{3}<-\frac{1}{3} .
$$

Hence,

$$
t_{2}^{m, k}-t_{0}^{m, k}>\frac{2 \pi}{n}-\frac{4}{n \mathcal{R}_{k}}+\frac{16}{3 n^{3} \mathcal{R}_{k}}+o\left(\frac{1}{\mathcal{R}_{k}}\right)
$$

and then

$$
\sum_{m=1}^{n}\left(t_{2}^{m, k}-t_{0}^{m, k}\right)>2 \pi-\frac{4}{\mathcal{R}_{k}}+\frac{16}{3 n^{2} \mathcal{R}_{k}}+o\left(\frac{1}{\mathcal{R}_{k}}\right)
$$

Therefore, by (3.11) we have, for $k$ large enough,

$$
\tau_{k}=\sum_{m=1}^{n}\left(t_{2}^{m, k}-t_{0}^{m, k}\right)+\sum_{m=1}^{n}\left(t_{4}^{m, k}-t_{2}^{m, k}\right)>2 \pi,
$$

which is a contradiction. Consequently, (3.12) holds.

Case 2: $\lambda_{0}>0$. Then there exists a constant $l_{0}>0$ such that, for $k$ large enough, $\lambda_{k} \geq l_{0}$. From (3.11) and the first equality of (3.2) we have, for $\theta_{k} \in\left(-\frac{\pi}{2}-2(m-1) \pi, \frac{\pi}{2}-2(m-1) \pi\right)$,

$$
t\left(\theta_{k}\right)=t_{0}^{1, k}+\frac{2}{n}\left(\frac{\pi}{2}-\theta_{k}\right)-\frac{2(m-1) \pi}{n}+o(1)
$$


Then, from the definition of $g\left(1+r_{k} \cos \theta_{k}, 1+r_{k}(t-\tau) \cos \theta_{k}(t-\tau), \lambda_{k}\right)$ we obtain

$$
\begin{gathered}
\int_{-\frac{\pi}{2}-2(m-1) \pi}^{\frac{\pi}{2}-2(m-1) \pi} g\left(1+r_{k} \cos \theta_{k}, 1+r_{k}(t-\tau) \cos \theta_{k}(t-\tau), \lambda_{k}\right) \cos \theta_{k} d \theta_{k} \\
\quad \leq \lambda_{k} \int_{-\frac{\pi}{2}-2(m-1) \pi}^{\frac{\pi}{2}-2(m-1) \pi}\left(g_{1}\left(1+r_{k}(t-\tau) \cos \theta_{k}(t-\tau)\right)-p(t)\right) \cos \theta_{k} d \theta_{k} \\
=-\frac{n \lambda_{k}}{2} \int_{t_{0}^{1, k}+\frac{2 m \pi}{n}+o(1)}^{t_{0}^{1, k}+\frac{2(m-1) \pi}{n}+o(1)}\left(g_{1}\left(x_{k}(t-\tau)\right)-p(t)\right) \cdot\left|\cos \left(\frac{\pi}{2}-\frac{n}{2} t\right)\right| d t \\
=\frac{n \lambda_{k}}{2} \int_{\frac{2(m-1) \pi}{n}}^{\frac{2 m \pi}{n}}\left(g_{1}\left(x_{k}(t-\tau)\right)-p(t)\right) \cdot\left|\cos \left(\frac{\pi}{2}-\frac{n}{2} t\right)\right| d t+o(1) .
\end{gathered}
$$

Denote

$$
I_{1}=\left[\frac{2(m-1) \pi}{n}, \frac{2 m \pi}{n}\right] \cap\left\{t: x_{k}(t-\tau) \geq 1\right\}
$$

and

$$
I_{0}=\left[\frac{2(m-1) \pi}{n}, \frac{2 m \pi}{n}\right] \cap\left\{t: x_{k}(t-\tau)<1\right\}
$$

Then $\operatorname{mes}\left(I_{0}\right)=o(1)$. Denote again

$$
I_{1}^{\prime}=\left[\frac{2(m-1) \pi}{n}-\tau, \frac{2 m \pi}{n}-\tau\right] \cap\left\{t: x_{k}(t) \geq 1\right\} .
$$

For $m=2 i-1, i=1,2, \ldots$, we have

$$
\begin{aligned}
\int_{\frac{2(m-1) \pi}{n}}^{\frac{2 m \pi}{n}} g_{1}\left(x_{k}(t-\tau)\right) \cdot\left|\cos \left(\frac{\pi}{2}-\frac{n}{2} t\right)\right| d t \\
=\int_{\frac{2(m-1) \pi}{n}}^{\frac{2 m \pi}{n}} g_{1}\left(x_{k}(t-\tau)\right) \cdot \sin \left(\frac{n}{2} t\right) d t \\
=\int_{I_{1}} g_{1}\left(x_{k}(t-\tau)\right) \cdot \sin \left(\frac{n}{2} t\right) d t+\int_{I_{0}} g_{1}\left(x_{k}(t-\tau)\right) \cdot \sin \left(\frac{n}{2} t\right) d t \\
=\int_{I_{1}} g_{1}\left(1+r_{k}(t-\tau) \cos \theta_{k}(t-\tau)\right) \cdot \sin \left(\frac{n}{2} t\right) d t+o(1) \\
=\int_{I_{1}} g_{1}\left(\mathcal{R}_{k} \sin \left(\frac{n}{2} t-\frac{n}{2} \tau\right)+O(1)\right) \cdot \sin \left(\frac{n}{2} t\right) d t+o(1) \\
=\int_{I_{1}^{\prime}} g_{1}\left(\mathcal{R}_{k} \sin \left(\frac{n}{2} t\right)+O(1)\right) \cdot \sin \left(\frac{n}{2} t+\frac{n}{2} \tau\right) d t+o(1) \\
=\cos \left(\frac{n}{2} \tau\right) \int_{I_{1}^{\prime}} g_{1}\left(\mathcal{R}_{k} \sin \left(\frac{n}{2} t\right)+O(1)\right) \cdot \sin \left(\frac{n}{2} t\right) d t \\
\quad+\sin \left(\frac{n}{2} \tau\right) \int_{I_{1}^{\prime}} g_{1}\left(\mathcal{R}_{k} \sin \left(\frac{n}{2} t\right)+O(1)\right) \cdot \cos \left(\frac{n}{2} t\right) d t+o(1) .
\end{aligned}
$$


Passing to limits, we get

$$
\lim _{k \rightarrow \infty} \int_{\frac{2(m-1) \pi}{n}}^{\frac{2 m \pi}{n}} g_{1}\left(x_{k}(t-\tau)\right) \cdot\left|\cos \left(\frac{\pi}{2}-\frac{n}{2} t\right)\right| d t=\frac{4}{n} \cos \left(\frac{n}{2} \tau\right) g(+\infty) .
$$

For $m=2 i, i=1,2, \ldots$, we similarly get

$$
\lim _{k \rightarrow \infty} \int_{\frac{2(m-1) \pi}{n}}^{\frac{2 m \pi}{n}} g_{1}\left(x_{k}(t-\tau)\right) \cdot\left|\cos \left(\frac{\pi}{2}-\frac{n}{2} t\right)\right| d t=\frac{4}{n} \cos \left(\frac{n}{2} \tau\right) g(+\infty) .
$$

Therefore we have

$$
\int_{\frac{2(m-1) \pi}{n}}^{\frac{2 m \pi}{n}} g_{1}\left(x_{k}(t-\tau)\right) \cdot\left|\cos \left(\frac{\pi}{2}-\frac{n}{2} t\right)\right| d t=\frac{4}{n} \cos \left(\frac{n}{2} \tau\right) g(+\infty)+o(1) .
$$

Consequently, we get

$$
\begin{aligned}
& \sum_{m=1}^{n} \int_{-\frac{\pi}{2}-2(m-1) \pi}^{\frac{\pi}{2}-2(m-1) \pi} g\left(1+r_{k} \cos \theta_{k}, 1+r_{k}(t-\tau) \cos \theta_{k}(t-\tau), \lambda_{k}\right) \cos \theta_{k} d \theta_{k} \\
& \quad \leq \sum_{m=1}^{n} \frac{n \lambda_{k}}{2} \int_{\frac{2(m-1) \pi}{n}}^{\frac{2 m \pi}{n}}\left(g_{1}\left(x_{k}(t-\tau)\right)-p(t)\right) \cdot\left|\cos \left(\frac{\pi}{2}-\frac{n}{2} t\right)\right| d t+o(1) \\
& \quad=\frac{n \lambda_{k}}{2}\left(4 \cos \left(\frac{n}{2} \tau\right) g(+\infty)-\int_{0}^{2 \pi} p(t)\left|\sin \left(\frac{n}{2} t+\theta\right)\right| d t\right)+o(1) .
\end{aligned}
$$

By $\left(\mathrm{H}_{2}^{\prime}\right)$ we obtain, for $k$ large enough,

$$
\sum_{m=1}^{n} \int_{-\frac{\pi}{2}-2(m-1) \pi}^{\frac{\pi}{2}-2(m-1) \pi} g\left(1+r_{k} \cos \theta_{k}, 1+r_{k}(t-\tau) \cos \theta_{k}(t-\tau), \lambda_{k}\right) \cos \theta_{k} d \theta_{k}<0
$$

Consequently, for $k$ large enough,

$$
\sum_{m=1}^{n}\left(t_{2}^{m, k}-t_{0}^{m, k}\right)>2 \pi-\frac{4}{\mathcal{R}_{k}}
$$

and then

$$
\tau_{k}>2 \pi,
$$

which also contradicts the $2 \pi$-periodicity of $\left(x_{k}(t), y_{k}(t)\right)$. This completes the proof.

Remark 3.5 Similarly, if $\left(\mathrm{H}_{1}\right),\left(\mathrm{H}_{2}^{\prime \prime}\right)$, and $\left(\mathrm{H}_{3}\right)$ hold, then the result in Lemma 3.4 is valid. Under these conditions, to estimate the maxima, we can we embed (1.1) into (2.2).

Lemma 3.6 Assume $\left(\mathrm{H}_{1}\right),\left(\mathrm{H}_{2}\right)$, and $\left(\mathrm{H}_{3}\right)$ hold. Then there exists a positive constant $M_{0}$ such that, for any $2 \pi$-periodic solution $x(t)$ of Eq. (2.1),

$$
\min _{t \in \mathbb{R}} x(t)>M_{0}
$$


Proof Let $\mathcal{R}_{k}$ be defined by (3.5). We first prove that there exists $c_{1}>0$ such that, for all $k \in \mathbb{N}^{+}$,

$$
x_{k}\left(t_{3}^{1, k}\right)>c_{1}
$$

Assume by contradiction that $x_{k}\left(t_{3}^{1, k}\right) \rightarrow 0$ (as $k \rightarrow \infty$ ). By Lemma 3.4 and Remark 3.5 there exist $M_{1}>0$ and $M_{2}>0$ such that

$$
\left\|x_{k}\right\|_{\infty}<M_{1}, \quad\left\|x_{k}^{\prime}\right\|_{\infty}<M_{2}
$$

Then $\mathcal{R}_{k}(t) \equiv c<+\infty$. However, by $\left(\mathrm{H}_{3}\right)$,

$$
\begin{aligned}
r_{k}^{2}\left(t_{3}^{1, k}\right)-r_{k}^{2}\left(t_{2}^{1, k}\right) & =\frac{8}{n^{2}} \int_{t_{2}^{1, k}}^{t_{3}^{1, k}} y_{k} g\left(x_{k}, x_{k}(t-\tau), \lambda_{k}\right) d t \\
& =\frac{8 \lambda_{k}}{n^{2}} \int_{t_{3}^{1, k}}^{t_{2}^{1, k}} y_{k} \frac{1}{x_{k}^{3}} d t-\left(1-\lambda_{k}\right) \frac{8}{n^{2}} \int_{t_{3}^{1, k}}^{t_{2}^{1, k}} y_{k} g_{0}\left(x_{k}\right) d t+O(1) \\
& =\frac{8 \lambda_{k}}{n^{2}} \cdot \frac{1}{x_{k}^{2}\left(t_{3}^{1, k}\right)}-\left(1-\lambda_{k}\right) \frac{8}{n^{2}} \int_{x\left(t_{3}^{1, k}\right)}^{1} g_{0}\left(x_{k}\right) d x_{k}+O(1) \\
& \rightarrow+\infty
\end{aligned}
$$

which is impossible. Therefore (3.14) holds. Similarly, we can obtain

$$
x_{k}\left(t_{3}^{m, k}\right)>c_{m}
$$

for $m=1,2, \ldots, n$. Consequently, we get the conclusion of Lemma 3.6.

Proof of Theorem 1.1 The result is obtained directly by Lemma 2.1, Lemma 3.4, Remark 3.5, and Lemma 3.6.

\section{Acknowledgements}

Research was supported by the National Natural Science Foundation of China (11401274, 11661046), Science and Technology Landing Project of Colleges and Universities in Jiangxi Province (KJLD14092), Science and Technology Project of Education Department of Jiangxi Province (GJJ151094), and Scientific Research Project of Jiujiang University (2013KJ18, 2014KJYB022)

\section{Competing interests}

The author declares that they have no competing interests.

\section{Author's contributions}

All authors read and approved the final manuscript.

\section{Publisher's Note}

Springer Nature remains neutral with regard to jurisdictional claims in published maps and institutional affiliations.

Received: 8 April 2017 Accepted: 13 June 2017 Published online: 25 July 2017

\section{References}

1. Bonheure, D, De Coster, C: Forced singular oscillators and the method of lower and upper solutions. Topol. Methods Nonlinear Anal. 22, 297-317 (2003)

2. Chu, J, Lin, X, Jiang, D, O'Regan, D, Agarwal, PR: Multiplicity of positive solutions to second order differential equations. Bull. Aust. Math. Soc. 73, 175-182 (2006) 
3. del Pino, MA, Manásevich, RF, Montero, A: T-Periodic solutions for some second order differential equations with singularities. Proc. R. Soc. Edinb., Sect. A 120, 231-243 (1992)

4. del Pino, MA, Manásevich, RF: Infinitely many T-periodic solutions for a problem arising in nonlinear elasticity. J. Differ. Equ. 103, 260-277 (1993)

5. Fonda, A, Manásevich, R, Zanolin, F: Subharmonic solutions for some second order differential equations with singularities. SIAM J. Math. Anal. 24, 1294-1311 (1993)

6. Fonda, A, Toader, R: Radially symmetric systems with a singularity and asymptotically linear growth. Nonlinear Anal. 7, 2485-2496 (2011)

7. Fonda, A, Garrione, M: A Landesman-Lazer type condition for asymptotically linear second order equations with singularity. Proc. R. Soc. Edinb., Sect. A 142, 1263-1277 (2012)

8. Habets, P, Sanchez, L: Periodic solution of some Liénard equations with singularities. Proc. Am. Math. Soc. 109 1135-1144 (1990)

9. Jiang, D, Chu, J, O'Regan, D, Agarwal, RP: Multiple positive solutions to superlinear periodic boundary value problems with repulsive singular forces. J. Math. Anal. Appl. 286, 563-576 (2003)

10. Jiang, D, Chu, J, Zhang, M: Multiplicity of positive periodic solutions to superlinear repulsive singular equations. J. Differ. Equ. 211, 282-302 (2005)

11. Lazer, AC, Solimini, S: On periodic solutions of nonlinear differential equations with singularities. Proc. Am. Math. Soc. 99, 109-114 (1987)

12. Li, J, Wang, Z: A Landesman-Lazer type condition for second-order differential equations with a singularity at resonance. Complex Var. Elliptic Equ. 60, 620-634 (2015)

13. $\mathrm{Ma}, T$, Wang, Z: Infinitely many periodic solutions of Duffing equations with singularities via time map. Abstr. Appl. Anal. 2014, Article ID 398512 (2014)

14. Wang, Z, Ma, T: Existence and multiplicity of periodic solutions of semilinear resonant Duffing equations with singularities. Nonlinearity 25, 279-307 (2012)

15. Wang, Z: Periodic solutions of Liénard equation with a singularity and a deviating argument. Nonlinear Anal., Real World Appl. 16, 227-234 (2014)

16. Wang, Z, Ma, T: Periodic solutions of Rayleigh equations with singularities. Bound. Value Probl. 2015, 154 (2015)

17. Zhang, M: A relationship between the periodic and the Dirichlet BVPs of singular differential equations. Proc. R. Soc. Edinb., Sect. A 128, 1099-1114 (1998)

18. Wang, Z: Lazer-Leach type conditions on periodic solutions of Liénard equation with a deviating argument at resonance. Abstr. Appl. Anal. 2013, Article ID 906972 (2013)

\section{Submit your manuscript to a SpringerOpen ${ }^{\circ}$ journal and benefit from:}

- Convenient online submission

- Rigorous peer review

Open access: articles freely available online

- High visibility within the field

- Retaining the copyright to your article

Submit your next manuscript at $\boldsymbol{~ s p r i n g e r o p e n . c o m ~}$ 